Review Article

\title{
What Is the de-qi-Related Pattern of BOLD Responses? A Review of Acupuncture Studies in fMRI
}

\author{
Jinbo Sun, ${ }^{1}$ Yuanqiang Zhu, ${ }^{1}$ Yang Yang, ${ }^{1}$ Lingmin Jin, ${ }^{1}$ \\ Karen M. von Deneen, ${ }^{1}$ Wei Qin, ${ }^{1}$ and Jie Tian ${ }^{1,2}$ \\ ${ }^{1}$ Life Sciences Research Center, School of Life Sciences and Technology, Xidian University, Xian, Shaanxi 710071, China \\ ${ }^{2}$ Institute of Automation, Chinese Academy of Sciences, Beijing 100190, China \\ Correspondence should be addressed to Wei Qin; wqin@ieee.org
}

Received 25 September 2012; Revised 24 December 2012; Accepted 6 January 2013

Academic Editor: Vitaly Napadow

Copyright (C) 2013 Jinbo Sun et al. This is an open access article distributed under the Creative Commons Attribution License, which permits unrestricted use, distribution, and reproduction in any medium, provided the original work is properly cited.

de-qi, comprising mostly subjective sensations during acupuncture, is traditionally considered as a very important component for the possible therapeutic effects of acupuncture. However, the neural correlates of $d e-q i$ are still unclear. In this paper, we reviewed previous fMRI studies from the viewpoint of the neural responses of de-qi. We searched on Pubmed and identified 111 papers. Fourteen studies distinguishing $d e-q i$ and sharp pain and eight studies with the mixed sensations were included in further discussions. We found that the blood oxygenation level-dependent (BOLD) responses associated with de-qi were activation dominated, mainly around cortical areas relevant to the processing of somatosensory or pain signals. More intense and extensive activations were shown for the mixed sensations. Specific activations of sharp pain were also shown. Similar BOLD response patterns between $d e$-qi evoked by acupuncture stimulation and $d e$-qi-like sensations evoked by deep pain stimulation were shown. We reckon that a standardized method of qualification and quantification of $d e-q i$, deeper understanding of grouping strategy of $d e-q i$ and sharp pain, and making deep pain stimulation as a control, as well as a series of improvements in the statistical method, are crucial factors for revealing the neural correlates of $d e-q i$ and neural mechanisms of acupuncture.

\section{Introduction}

Acupuncture, as a key component of traditional Chinese medicine (TCM) and an alternative and complementary therapy in western society, has been widely used all over the world. As an old concept that appeared in a very early Chinese classic text, de-qi was believed to be an indispensable component of acupuncture treatment [1]. In recent years, the importance of $d e-q i$ has been investigated based on the modern biological and medical framework [2-14]. Several studies reported the correlation between the de-qi sensations and analgesic effects $[2-4,10-12]$ or the electroencephalogram changes [9], whereas others do not $[8,13,14]$. Therefore, the role of de-qi in acupuncture treatment is still controversial $[1,5,6,15]$.

Many researchers have begun to qualify and quantify sensations of de-qi [15-27], because they argued that the lack of a competent measure of de-qi affected the association between $d e-q i$ and the clinical outcomes $[1,18,19]$. Most studies reported that $d e-q i$ sensations involved numbness, heaviness, aching, dull pain, and tingling $[1,16,18,19,23$, $25,27]$. Other sensations such as fullness/distention, soreness, and pressure were also included in part of the studies $[1,16$, $19,27]$. The dimensions of sensations of de-qi were largely different, which ranged from four [19] to seventeen [25]. Although there have not a consensus that which sensations were involved in $d e-q i$, researchers focused on qualifying and quantifying sensations of $d e-q i$, and they agreed that deqi includes specific sensations for acupuncture stimulation $[1,16,18,27]$. Besides, another consensus was reached that as a conventional component of the needling sensations presented during acupuncture manipulation, sharp pain is noxious and is what acupuncturists try to avoid during needle manipulation $[1,16,18,25]$. It is often excluded from the 
components of de-qi and regarded as the sensation that is irrelevant to the acupuncture effect $[1,16,18,25]$.

With the aid of functional magnetic resonance imaging (fMRI) techniques, it is possible to explore the neural responses of $d e-q i$ and further interpret the neural mechanisms of acupuncture. More than one hundred studies have been published to explore the neurobiological mechanisms of acupuncture in the past few decades [28-36]. However, few of them paid close attention to the neural correlation of de-qi. Therefore, several fundamental issues about the central responses of $d e-q i$ remain open to debate. First of all, although researchers agreed that de-qi has specific sensations for acupuncture stimulation [16], the sensory quality of $d e-q i$ was similar to that of deep pain constructed by intramuscular injections of hypertonic saline, which mainly included tenderness, heaviness, aching, cramping, throbbing, and gnawing $[25,37]$. Thus, the quality of the sensation of de$q i$ was likely nonspecific $[38,39]$. Up to now, however, direct comparisons of blood oxygenation level-dependent (BOLD) responses between de-qi sensations evoked by acupuncture stimulation and de-qi-like sensations evoked by deep pain stimulation have not yet been investigated. Only one recent study involved the indirect comparisons of these two stimulations, which had a similar sensory quality, in the discussion [40]. Secondly, in the domain of pain studies, it is generally known that differences in the quality and origin between sharp pain and deep pain are remarkable [41]. Abundant evidence from animal investigations [42, 43], clinical data [44-46], and human neuroimage studies [37, 4752] indicated different brain processing pathways for acute superficial pain (sharp pain) and deep pain. The sharp pain following acupuncture stimulation is sometimes perceived by subjects. However, most previous acupuncture fMRI studies did not explicitly distinguish the needle sensations into de-qi and sharp pain. To make matters worse, significant incompatibilities were shown across the results of several studies which excluded subjects who experienced sharp pain $[22,23,25]$ or divided subjects into two groups according to whether subjects experienced sharp pain during acupuncture manipulation [33, 53-55].

In this paper, we would like to summarize the results of fMRI-based acupuncture studies from the perspective of the general pattern of central BOLD responses of de-qi. We aim to organize the evidence about three fundamental questions. First, what is the pattern of central responses of $d e$-qi evoked by during acupuncture stimulation? Second, how are the distinct patterns of central BOLD responses associated with de-qi and sharp pain? Thirdly, are the patterns of central BOLD responses associated with de-qi specific from those of deep pain in pain studies? In addition, we hope to offer several suggestions for future studies of the neural correlates of $d e-q i$ evoked by acupuncture stimulation.

\section{Identification of the Relevant Literature}

The acupuncture studies involved in this paper were first identified by searching on Pubmed using the key words "acupuncture" or "electroacupuncture" in the title and
"fMRI" or "functional magnetic resonance imaging" in the title/abstract (four combinations of key words: "acupuncture" and "fMRI," "acupuncture" and "functional magnetic resonance imaging," "electroacupuncture" and "fMRI," and "electroacupuncture" and "functional magnetic resonance imaging"). About 111 original studies published in peerreviewed journals in English were included in the search (two studies in the Evidence-Based Complementary and Alternative Medicine with the state of "in press" were also involved). Firstly, 17 review articles were excluded. Secondly, 23 studies which did not evaluate and record subjects' needling sensations were discarded from further discussions. Thirdly, 13 studies that were based on data-driven methods were excluded. Fourthly, 4 studies that aimed to explore the continuous effect of acupuncture were also excluded. Fifthly, other 15 studies were also not included because of the lack of $d e$-qi-related BOLD responses ( 2 were behavioral studies, 2 were magnetoencephalogram-based studies, 4 studies were associated with the therapeutic/placebo effect of acupuncture, 1 study was laser-acupuncture-based, and 5 studies lacked the whole brain's one-sample $t$-test results). In the remaining 39 studies, 17 studies asked or evaluated the subjects' de-qi but did not mention whether subjects underwent sharp pain $[36,38,56-70]$. Eight studies which asked or evaluated the subjects' de-qi and sharp pain but did not distinguish $d e-q i$ and sharp pain in the fMRI analysis [53, 71-77] were named as "mixed pattern studies" and summarized in Table 3. Fourteen studies that excluded subjects who experienced sharp pain for the fMRI data analysis [32, 34, 78-85] or divided subjects into two groups according to whether subjects experienced sharp pain during acupuncture manipulation $[33,40,54,55]$ were named as "pure de-qi pattern studies" and are summarized in Table 2. The experimental details and the methodological details of the 14 de-qi-related studies are shown in Table 1 and Table 5, respectively.

\section{General Observations}

Reviewing the studies of acupuncture in fMRI, we found that de-qi and sharp pain were not distinguished in most studies (97 out of 111, 87\%). Therefore, limited resources could be used for summarizing the de-qi BOLD response patterns. We suggest that it is necessary to differentiate $d e-q i$ and sharp pain in further fMRI acupuncture studies. In the following part, we will summarize the BOLD response patterns of pure de-qi, the BOLD response patterns of mixed sensations, the similarities/differences of BOLD response patterns between pure $d e-q i$ and mixed sensations, similarities/differences of BOLD response patterns between pure $d e-q i$ and $d e$-qi-like sensations evoked by deep pain stimulation, and the comparison of $d e$-qi-related regions with regions generally activated in acupuncture fMRI studies, which were summarized in other reviews $[39,86]$.

3.1. The BOLD Responses Pattern of Pure de-qi. The common activations (frequency $>4$ or $29 \%$ ) of de-qi were the SI, SII, thalamus, MI, cerebellum, insula, inferior parietal lobe, and 
TABLE 1: Experimental details of fMRI studies on BOLD responses of de-qi.

\begin{tabular}{|c|c|c|c|c|}
\hline Studies & Acupoints used & Stimulation & $\begin{array}{c}\text { Sensations } \\
\text { evaluated }\end{array}$ & Grouping \\
\hline \multicolumn{5}{|c|}{ Studies that compared central BOLD responses between $d e-q i$ and sharp pain groups } \\
\hline Hui et al., 2000 [54] & LI4 & Manual & de-qi, pain & de-qi group: 11; sharp pain group: 2 \\
\hline Hui et al., 2005 [33] & ST36 & Manual & Their own scale & de-qi group: 11; sharp pain group: 4 \\
\hline Hui et al., 2009 [55] & LI4, LV3, ST36 & Manual & Their own scale & de-qi group: 37 (scans); sharp pain group: 29 (scans) \\
\hline Sun et al., 2012 [40] & ST36 & Manual & Their own scale & de-qi group: 19; sharp pain group: 19 \\
\hline \multicolumn{5}{|c|}{ Studies where no subjects reported sharp pain/excluded subjects who experienced sharp pain from fMRI analysis } \\
\hline $\begin{array}{l}\text { Napadow et al., } 2005 \\
{[34]}\end{array}$ & ST36 & Electro & Their own scale & Total: 13 \\
\hline Jeun et al., 2005 [78] & GB34 & Manual & Their own scale & Total: 10 \\
\hline Kong et al., 2007 [79] & GB37, UB60 & Electro & SASS & Total: 8 \\
\hline Zhou and Jia, 2008 [66] & HT7, ST36, ST40, KI3 & Electro & Their own scale & Total: 26 \\
\hline Fang et al., 2009 [32] & LV3, LV2, ST44 & Manual & Their own scale & Total: 10 \\
\hline Na et al., 2009 [80] & GB34 & Manual & Their own scale & Total: 12 \\
\hline Bai et al., 2010 [81] & PC6, PC7, GB37 & Electro & Their own scale & Total: 36 \\
\hline Qiu et al., 2010 [82] & LI4, LV3, ST36 & Manual & Their own scale & Total: 38 \\
\hline Fang et al., 2012 [85] & CV4, CV12 & Electro & Their own scale & Total: 17 \\
\hline Sun et al., 2012 [40] & ST36 & Manual & Their own scale & Total: 52 \\
\hline
\end{tabular}

anterior MCC. The common deactivations of de-qi were the ACC, amygdala, hippocampus, parahippocampus, hypothalamus, temporal pole, and PCC. Most of the commonly responding regions (11/15 or $73 \%$ ) were divergent across studies.

3.2. The BOLD Response Patterns of Mixed Sensations. The common activations (frequency $>2$ or $25 \%$ ) of mixed sensations were the SI, SII, MI, cerebellum, SMA, insula, IMG, DLPFC/VLPFC, and pMCC. The common deactivations of de-qi were the STG, inferior temporal gyrus, precuneus, lingual gyrus, occipital gyrus, and PCC.

3.3. The Similarities/Differences of BOLD Response Patterns between Pure de-qi and Mixed Sensations. In the 14 "pure deqi pattern studies," only two studies performed the betweengroup analysis that statistically compared the de-qi group and mixed group (de-qi + sharp pain) based on a twosample unpaired $t$-test $[40,55]$. In Hui et al., 2009, significant differences in the posterior cingulate/precuneus, pregenual cingulate/frontal pole, subgenual area, orbitofrontal cortex, temporal pole, amygdala, hippocampus, parahippocampus, hypothalamus, cerebellar vermis (lobules VII and VIII), SII, and anterior middle cingulate were shown between the de-qi group and mixed group. Specifically, activity of BOLD signals was significantly less activated or more deactivated in the de$q i$ group than that in the mixed group. In Sun et al., 2012, significantly stronger activations of the mixed group were presented in the bilateral putamen, the bilateral thalamus, and the bilateral cerebellum (CrusI and CrusII) [40]. In these regions, BOLD signals of the de-qi group were barely changed, while significant BOLD responses were shown in the mixed group.
3.4. The Similarities/Differences of BOLD Response Patterns between Pure de-qi and de-qi-Like Sensations Evoked by Deep Pain Stimulation. In contrast to superficial (cutaneous) pain, deep pain (originating from muscle, joints or viscera) is dull, diffuse, and difficult to localize $[37,50]$. In Henderson et al., 2006, the researchers used intramuscular injections of hypertonic saline to construct a deep pain model [37]. The subjective sensations under this deep pain mainly included tenderness, heaviness, aching, cramping, throbbing, and gnawing, which were similar to that of de-qi evoked by acupuncture stimulation. Table 4 summarizes the activations and deactivations from the deep pain studies [37, 48, 50, $87,88]$. Common activations (frequency $>2$ or $40 \%$ ) were seen in the anterior cingulate, posterior cingulate, SI, SII, MI, insula, cerebellar cortices, inferior parietal, claustrum, and thalamus. A common deactivation was in the perigenual cingulate. Most of the activations for deep pain were consistent with that of $d e$-qi evoked by acupuncture stimulation, except for the anterior cingulate and posterior cingulate, which were commonly deactivated during de-qi. Besides, the amygdala, hippocampus, parahippocampus, hypothalamus, and temporal pole were deactivated for de-qi but not for deep pain.

3.5. The Comparison of de-qi-Related Regions with Regions Generally Activated in Acupuncture fMRI Studies. Huang et al., 2012, summarized the general BOLD responses in acupuncture fMRI studies [86]. The supramarginal gyrus/insula/SII, presupplementary motor area/middle cingulate, thalamus, and precentral gyrus were the most commonly found activations. Besides, the anterior cingulate, subgenual cortex, amygdala/hippocampal formation, ventromedial prefrontal cortex, and posterior cingulate were 
TABLE 2: de-qi-related BOLD responses evoked by acupuncture stimulation.

\begin{tabular}{|c|c|c|}
\hline \multirow{2}{*}{ Regions } & \multicolumn{2}{|c|}{ (Reference no.) } \\
\hline & Activations & Deactivations \\
\hline \multicolumn{3}{|l|}{ Somatosensory system } \\
\hline SI & {$[32,34,40,54,79,84]$} & {$[79,82]$} \\
\hline SII & {$[32-34,54,55,79,85]$} & \\
\hline Thalamus & {$[32,34,40,80,83-85]$} & {$[33]$} \\
\hline Caudate & {$[80]$} & {$[33,54]$} \\
\hline Putamen & {$[34,40,80]$} & {$[54]$} \\
\hline Brainstem & [33] & {$[33,34]$} \\
\hline \multicolumn{3}{|l|}{ Motor system } \\
\hline M1 & {$[40,78,78,80,83]$} & {$[33]$} \\
\hline Cerebellum & {$[40,79-81,83,84]$} & {$[33,55,82]$} \\
\hline SMA & {$[32,85]$} & \\
\hline \multicolumn{3}{|l|}{ Sensory integration system } \\
\hline Insula & {$[32,34,40,79,81-85]$} & {$[33,54]$} \\
\hline ACC & & {$[33,54,55,85]$} \\
\hline \multicolumn{3}{|l|}{ Special senses } \\
\hline IFG & {$[40,83]$} & \\
\hline STG & {$[40,83,84]$} & \\
\hline \multicolumn{3}{|l|}{ MTG } \\
\hline \multicolumn{3}{|l|}{ Frontal cortex } \\
\hline vmPFC & {$[82]$} & {$[33,34,85]$} \\
\hline Frontal Pole & {$[34]$} & {$[32,33,55]$} \\
\hline Superior frontal gyrus & {$[80]$} & [79] \\
\hline Medial orbital prefrontal cortex & & {$[79]$} \\
\hline Medial frontal gyrus & & {$[79]$} \\
\hline Ventromedial frontal pole & & {$[34]$} \\
\hline Dorsomedial frontal pole & & {$[32,34]$} \\
\hline DLPFC/VLPFC & {$[34]$} & \\
\hline DMPFC & {$[34]$} & \\
\hline OFC/MPFC & {$[34]$} & {$[82]$} \\
\hline Paracentral lobule & {$[33]$} & {$[79]$} \\
\hline Pregenual cingulate cortex & & {$[82]$} \\
\hline \multicolumn{3}{|l|}{ Subcortical gray matter } \\
\hline N. accumbens/septi & & {$[33,54]$} \\
\hline Amygdala & {$[81]$} & {$[32-34,54,55,82]$} \\
\hline Hippocampus & {$[84]$} & {$[32,33,54,55,82]$} \\
\hline Parahippocampus & {$[34]$} & {$[32,33,54,55,79,82]$} \\
\hline Hypothalamus & {$[79,84]$} & {$[33,54,55,79]$} \\
\hline Ventral tegmental area & & {$[54]$} \\
\hline Retrosplenial cortex & & {$[33]$} \\
\hline Lentiform nucleus & {$[80]$} & \\
\hline Claustrum & {$[40,80,83]$} & \\
\hline PAG & {$[81,84,85]$} & \\
\hline Nucleus raphe & [34] & \\
\hline Midbrain & {$[83]$} & \\
\hline \multicolumn{3}{|l|}{ Temporal/parietal cortex } \\
\hline Temporal pole & & {$[32,33,54,55,82]$} \\
\hline Inferior parietal lobe & {$[34,40,79-81,83]$} & {$[33]$} \\
\hline Precuneus & {$[83]$} & {$[32,82]$} \\
\hline Middle temporal gyrus & {$[84]$} & [79] \\
\hline Superior parietal lobule & {$[78,83,84]$} & {$[79]$} \\
\hline Transverse temporal gyrus & {$[40,84]$} & \\
\hline
\end{tabular}


TABLE 2: Continued.

\begin{tabular}{lcc}
\hline Regions & Activations & (Reference no.) \\
\hline Occipital cortex & & \\
$\quad$ Lingual gyrus & & {$[79]$} \\
Cuneus & & {$[32]$} \\
Occipital gyrus & & {$[81]$} \\
Retrosplenial cortex & {$[84]$} \\
Angular gyrus & {$[84]$} \\
Fusiform gyrus & {$[82]$} \\
Middle occipital gyrus & {$[34]$} \\
Cingulate gyrus & {$[82]$} \\
PCC & {$[32,34,40,83]$} \\
pMCC & {$[32]$} \\
Subgenual cingulate & {$[34]$} \\
aMCC & {$[32,33,55,82]$} \\
\hline
\end{tabular}

the most common deactivations. Beissner, 2011, summarized the common BOLD responses evoked by acupuncture stimulation based on studies which met the methodological inclusion criteria [39]. The most frequently activated cortical areas were the SII, insula, SI, cerebellum, thalamus, MI, STG, visual cortices, IFG, SMA/pre-SMA, basal ganglia, MTG, and ACC. Significant differences in the regions generally activated in acupuncture fMRI studies were shown between these two previous reviews. When comparing our results with theirs, we found that our results were more similar to the results of Huang et al., 2012, but were more extensive (particularly showing more deactivations) than the results of Beissner, 2011. We argued that it was because our review of de-qirelated patterns and results of Huang et al., 2012, lacked the methodological inclusion criteria [39].

\section{Discussion}

This paper summarized the results of fMRI-based acupuncture studies from the perspective of the general pattern of central BOLD responses of de-qi. Three fundamental issues are discussed later. In addition, several suggestions for future studies of the neural correlates of de-qi evoked by acupuncture stimulation were offered.

4.1. Activation-Dominated BOLD Responses Associated with de-qi during Acupuncture Stimulation. The most robust BOLD responses evoked by acupuncture stimulation were in the SII, insula, SI, cerebellum, thalamus, MI, STG, visual cortices, IFG, SMA/pre-SMA, basal ganglia, MTG, and ACC. Under the methodological inclusion criteria [39], only the deactivation of the occipital cortex was shown. For the de$q i$-related BOLD responses, a similar activation pattern was shown, including in the SI, SII, thalamus, MI, cerebellum, insula, inferior parietal lobe, and anterior MCC. Besides, several deactivations were seemingly the common pattern of de-qi. However, we argued that the reliability of these deactivations was poor. We inferred that several possible reasons might contribute to these deactivation patterns. Firstly, the average of the repeated runs for each subject was applied. Repetition may itself alter the distribution of the activated regions due to the influence of memory and expectation [48]. Secondly, global normalization, a questionable data processing step adopted in several fMRI-based acupuncture studies, can introduce an artificially negative relationship with the task [83, 89-92]. Particularly, our recent study clarified that for the fMRI-based acupuncture data, global normalization significantly changed the activation-dominated results to deactivation-dominated ones [83]. Therefore, we suggested that BOLD responses associated with de-qi during acupuncture stimulation should be activation dominated.

4.2. Distinct Patterns of Central BOLD Responses Associated with de-qi and Sharp Pain. Since subjects with pure sharp pain during acupuncture stimulation were difficult to obtain, the differences between $d e-q i$ and sharp pain evoked by acupuncture stimulation could not be presented directly. Therefore, we had to infer their differences between the de-qi and mixed sensations. However, studies which were statistically compared with the BOLD responses between de-qi and mixed sensations were also a scarcity [40, 55]. Our recent study focused on these issues and indicated that both the quantitative and qualitative differences of BOLD responses between $d e-q i$ and mixed sensations evoked by acupuncture stimulation were distinct [40]. We inferred that the pattern of BOLD responses for sharp pain might be partly separated from that of $d e-q i$ in the spatial distribution. The subjects with sharp pain should be excluded from those with only $d e-q i$ when exploring the central BOLD responses during acupuncture stimulation [40].

4.3. Similar BOLD Response Patterns between de-qi Evoked by Acupuncture Stimulation and de-qi-Like Sensations Evoked by Deep Pain Stimulation. Most common activations of de$q i$ in this paper were shown in the aforementioned deep pain studies, which might indicate similar central processing of the same origin of stimulation and similar subjective 
TABLE 3: mixed-related BOLD responses evoked by acupuncture stimulation.

\begin{tabular}{|c|c|c|}
\hline \multirow{2}{*}{ Regions } & \multicolumn{2}{|c|}{ (References no.) } \\
\hline & Activations & Deactivations \\
\hline \multicolumn{3}{|l|}{ Somatosensory system } \\
\hline SI & {$[71,75]$} & {$[75]$} \\
\hline SII & {$[72,75,76]$} & \\
\hline thalamus & [77] & {$[53]$} \\
\hline Supermarginal & {$[76]$} & \\
\hline putamen & {$[71]$} & {$[71]$} \\
\hline brainstem & {$[77]$} & {$[77]$} \\
\hline \multicolumn{3}{|l|}{ Motor system } \\
\hline M1 & {$[71,75,76]$} & {$[74]$} \\
\hline cerebellum & {$[53,72,74]$} & \\
\hline SMA & {$[75,77]$} & \\
\hline \multicolumn{3}{|l|}{ Sensory integration system } \\
\hline insula & {$[53,71,75-77]$} & {$[71]$} \\
\hline ACC & & {$[77]$} \\
\hline \multicolumn{3}{|l|}{ Special senses } \\
\hline IFG & {$[75,76]$} & \\
\hline STG & {$[75]$} & {$[53,71]$} \\
\hline MTG & & [53] \\
\hline \multicolumn{3}{|l|}{ Frontal cortex } \\
\hline vmPFC & & {$[77]$} \\
\hline Frontal Pole & & {$[74]$} \\
\hline Frontal operculum & {$[75]$} & \\
\hline Medial orbital prefrontal cortex & & {$[72]$} \\
\hline Medial frontal gyrus & & {$[74]$} \\
\hline DLPFC/VLPFC & {$[75,76]$} & {$[75]$} \\
\hline DMPFC & {$[75]$} & \\
\hline Paracentral lobule & & {$[77]$} \\
\hline Amygdala & {$[76]$} & {$[77]$} \\
\hline Hippocampus & {$[76]$} & \\
\hline Parahippocampus & {$[53]$} & \\
\hline Lentiform nucleus & & {$[71]$} \\
\hline PAG & & {$[76]$} \\
\hline \multicolumn{3}{|l|}{ Temporal/parietal cortex } \\
\hline Inferior temporal gyrus & & {$[72,74]$} \\
\hline Inferior parietal lobe & {$[72]$} & {$[75]$} \\
\hline Precuneus & & {$[71,74,75,77]$} \\
\hline Superior parietal lobule & {$[75]$} & \\
\hline traisverse temporal gyrus & {$[72]$} & \\
\hline \multicolumn{3}{|l|}{ Occipital cortex } \\
\hline Lingual gyrus & & {$[53,75]$} \\
\hline Cuneus & {$[75]$} & \\
\hline Occipital gyrus & & {$[53,72,74]$} \\
\hline angular gyrus & {$[75]$} & \\
\hline fusiform gyrus & & {$[53]$} \\
\hline \multicolumn{3}{|l|}{ Cingulate gyrus } \\
\hline PCC & & {$[71,75,77]$} \\
\hline pMCC & {$[76,77]$} & \\
\hline
\end{tabular}

sensations. Due to individual variability of brain morphology and differences in experimental design, the central patterns of activation during deep pain and acupuncture stimulation were difficult to compare between studies. Therefore, we suggested that a specific central effect of de-qi during acupuncture stimulation might be illustrated after comparing it directly to deep pain stimulation.

4.4. Several Suggestions for Future Studies of the Neural Correlates of de-qi Evoked by Acupuncture Stimulation. First of all, for better understanding what de-qi is and exploring its possible role in acupuncture, many researchers have been engaged in qualifying and quantifying de-qi [1, 15-27]. However, in the aforesaid fMRI-based acupuncture studies about de-qi, different sensation questionnaires were used to quantify subjects' acupuncture sensations (see Table 1 for details). This may lead to two results. One is that the kinds of acupuncture sensations recorded by different studies are partially different. The other is that the same score of an acupuncture sensation between different studies refers to a different subjective intensity experienced by subjects due to the various definitions of the same score between different studies. Thus, acupuncture sensations recorded by different studies are inappropriate to compare. We suggest that further studies should pay more attention to the quantification questionnaire of $d e-q i$ and try to apply a standardized quantification method to better control the experimental conditions $[38,76]$.

Secondly, another important discrepancy among studies is the definition of grouping. In Hui et al. and Asghar et al. $[33,53]$, they both grouped subjects into two groups (the de$q i$ group and mixed group). But the definition of the "deqi group" was totally different in the two studies. In the studies of Hui, the de-qi group referred to subjects who only experienced de-qi during acupuncture, while in Asghar et al., the de-qi group referred to subjects whose de-qi scores were greater than sharp pain, which were similar to the Sun et al's sharp pain group (de-qi sensation mixed with sharp pain). Sun et al's study indicated that even a little sharp pain mixed in, both the quantitative and qualitative differences of BOLD responses between de-qi and mixed sensations evoked by acupuncture stimulation were significant [40]. Therefore, we proposed that subjects with sharp pain should be separated from those with only de-qi when exploring the central BOLD responses during acupuncture stimulation. Further comparisons between de-qi and sharp pain should be accumulated. Besides, the deep pain stimulations, which had a similar sensory quality to de-qi evoked by acupuncture stimulation, could serve as a valid control for acupuncture.

At last, methodological problems and differences may partly contribute to the discrepancies among studies which were reviewed here. Beissner and Henke found that most acupuncture studies lacked adequate strict statistical methods and suggested that several methodological problems should be solved to facilitate acupuncture studies [93]. The improvements for obtaining more reliable results included a larger sample size, corrected threshold, and a more robust method of statistical inference [94]. With regards to the studies reviewed in this paper, an inappropriate group analysis 
TABLE 4: BOLD responses evoked by deep pain stimulation.

\begin{tabular}{|c|c|c|c|c|c|}
\hline & $\begin{array}{l}\text { Henderson et al., } \\
2006 \text { [37] }\end{array}$ & $\begin{array}{c}\text { Henderson et al., } \\
2008 \text { [48] }\end{array}$ & $\begin{array}{c}\text { Macefield et al., } \\
2007 \text { [87] }\end{array}$ & $\begin{array}{c}\text { Niddam et al., } 2002 \\
{[50]}\end{array}$ & $\begin{array}{c}\text { Maeda et al., } 2011 \\
{[88]}\end{array}$ \\
\hline anterior cingulate & & $\uparrow$ & $\uparrow$ & $\uparrow$ & $\uparrow$ \\
\hline posterior cingulate & & $\uparrow$ & $\uparrow$ & $\uparrow$ & $\uparrow$ \\
\hline mid-cingulate & $\uparrow$ & & & & \\
\hline cingulate motor area (CMA) & $\uparrow$ & & & & \\
\hline primary somatosensory (S1) & $\uparrow$ & $\uparrow$ & $\uparrow$ & $\uparrow$ & \\
\hline secondary somatosensory (SII) & & $\uparrow$ & $\uparrow$ & $\uparrow$ & $\uparrow$ \\
\hline motor cortices (M1) & $\uparrow$ & & & $\uparrow$ & \\
\hline anterior insular & $\uparrow$ & & $\uparrow$ & $\uparrow$ & \\
\hline mid-insular & $\uparrow$ & $\uparrow$ & & & $\uparrow$ \\
\hline posterior insular & $\uparrow$ & & $\uparrow$ & $\uparrow$ & \\
\hline cerebellar cortices & & $\uparrow \downarrow$ & $\uparrow$ & & \\
\hline Inferior frontal & & & & $\uparrow$ & \\
\hline Medial frontal & & & & $\uparrow$ & \\
\hline Middle frontal & & & & $\uparrow$ & \\
\hline Superior temporal & & & & $\uparrow$ & \\
\hline Inferior parietal & & & & $\uparrow$ & $\uparrow$ \\
\hline Claustrum & & & & $\uparrow$ & $\uparrow$ \\
\hline Thalamus & & & & $\uparrow$ & $\uparrow$ \\
\hline Precuneus & & & & $\uparrow$ & \\
\hline putamen & & & & & $\uparrow$ \\
\hline caudate & & & & & $\uparrow$ \\
\hline prefrontal (DLPFC) & & $\downarrow$ & & & $\uparrow$ \\
\hline perigenual cingulate (PACC) & $\downarrow$ & $\downarrow$ & $\downarrow$ & & \\
\hline hippocampus & & $\downarrow$ & & & \\
\hline
\end{tabular}

method, a too small sample size, and the liberal threshold applied by the studies could be the most important problems (see Table 3 for details). For instance, first, Hui et al. [33] applied the fixed-effect model in the group analysis, which could produce positive group results even when only a single subject has strong activations [93]. Second, Hui et al. recruited 13 subjects in their study, and only two subjects experienced the mixed sensation [54]. Hui et al. recruited 15 subjects and a mixed group with 4 subjects [33]. Such a sample size ( 2 subjects and 4 subjects) is too small to obtain group results with sufficient statistical power. Third, several studies adopted global normalization $[33,53,55]$, which is a questionable data processing step and can introduce an artificially negative relationship with the task [89-92, 95-98]. Particularly, our recent study clarified that for the fMRI-based acupuncture data, global normalization significantly changed the activation-dominated results to deactivation-dominated ones [83]. As a whole, solving these crucial methodological problems could greatly help studies on neural correlates of $d e-q i$ to obtain more repeatable and reliable results. Thus, we suggest that future studies should focus on improvements in their methodological problems which we believe could shed light on studies of neural correlates of de-qi.

\section{Synopsis and Possible Solutions}

de-qi, comprising mostly of subjective sensations during acupuncture, is traditionally considered as a very important component of the possible therapeutic effects of acupuncture. Thus, it is of great importance to reveal the neural correlates of de-qi which may benefit the understanding of neural mechanisms of acupuncture. However, previous studies in fMRI involving the BOLD response of $d e-q i$ were limited and did not reach consistent conclusions on the neural response pattern of $d e-q i$ in the brain. In this paper, we summarized previous fMRI studies on the neural responses of $d e-q i$ and answered three fundamental questions. For the first question concrning the pattern of the central responses of $d e$-qi evoked by acupuncture stimulation, our answer was that the BOLD responses associated with $d e-q i$ during acupuncture stimulation were activation dominated, mainly around cortical areas relevant to the processing of somatosensory or pain signals. For the second question on how the distinct patterns of central BOLD responses are associated with de-qi and sharp pain, our answer was that more intensive and extensive activations were shown for the mixed sensations evoked by acupuncture stimulation. Specific activations of sharp pain 
TABLE 5: Methodological details of fMRI studies on BOLD responses for de-qi.

\begin{tabular}{|c|c|c|c|c|}
\hline Studies & One group analysis method & $P$ value of a one group analysis & $\begin{array}{l}\text { Two group analysis } \\
\text { method }\end{array}$ & $\begin{array}{l}P \text { value of a two } \\
\text { group analysis }\end{array}$ \\
\hline \multicolumn{5}{|c|}{ Studies that compared central BOLD responses between $d e-q i$ and sharp pain groups } \\
\hline Hui et al., 2000 [54] & Kolmogorov-Smirnov test & $P<0.001$ or $P<0.00001$ (cor) & & \\
\hline Hui et al., 2005 [33] & group-averaged & $P<0.001$ (unc), minimum 3 voxels & & \\
\hline Hui et al., 2009 [55] & group-averaged & $P<0.001$ (unc), minimum 3 voxels & $\begin{array}{l}\text { two-sample } \\
\text { unpaired } t \text {-tests }\end{array}$ & $P<0.05$ (unc) \\
\hline Sun et al., 2012 [40] & random effect model & $P<0.0001$ (unc), minimum 5 voxels & $\begin{array}{c}\text { two-sample } \\
\text { unpaired } t \text {-tests }\end{array}$ & $P<0.0001$ (unc) \\
\hline \multicolumn{5}{|c|}{ Studies where no subjects reported sharp pain/excluded subjects who experienced sharp pain from fMRI analysis } \\
\hline Napadow et al., 2005 [34] & Fixed effect model & $P<0.0001$ (unc), minimum 3 voxels & & \\
\hline Jeun et al., 2005 [78] & random effect model & $P<0.01($ unc) & & \\
\hline Kong et al., 2007 [79] & random effect model & $P<0.05$ (cor), minimum 10 voxels & & \\
\hline Zhou and Jia 2008 [66] & random effect model & $P<0.001$ (cor) 50 voxels & & \\
\hline Na et al., $2009[80]$ & random effect model & $P<0.001$ (unc), minimum 100 voxels & & \\
\hline Bai et al., 2010 [81] & random effect model & $P<0.005$ (unc), minimum 5 voxels & & \\
\hline Qiu et al., 2010 [82] & random effect model & $P<0.001$ (unc), minimum 3 voxels & & \\
\hline Fang et al., 2012 [85] & random effect model & $P<0.05$ (cor), minimum 5 voxels & & \\
\hline Sun et al., 2012 [40] & random effect model & $P<0.0001$ (unc), minimum 5 voxels & & \\
\hline
\end{tabular}

were also shown. For the third question asking if the patterns of central BOLD responses were associated with de-qi specifically from those of deep pain in pain studies, our answer was that similar BOLD response patterns between de-qi evoked by acupuncture stimulation and de-qi-like sensations evoked by deep pain stimulation were shown. Finally, we reckon that a standardized method of qualification and quantification of $d e-q i$, a deeper understanding of grouping strategy of $d e-q i$ and sharp pain, and marking the deep pain stimulation as a control, as well as a series of improvements in the statistical method, are crucial factors for revealing the neural correlates of $d e-q i$ and neural mechanisms of acupuncture.

\section{Conflict of Interests}

The authors declare that they have no conflict of interests.

\section{Acknowledgments}

This study was supported by the Project for the National Key Basic Research and Development Program (973) under Grant nos. 2012CB518501 and 2011CB707702, the National Natural Science Foundation of China under Grant nos. 30930112, 30970774, 81000640, 81000641, 81030027, 81101036, 81101108, 31150110171, 30901900, 81271644, and 31200837, and the Fundamental Research Funds for the Central Universities.

\section{References}

[1] J. Kong, R. Gollub, T. Huang et al., "Acupuncture De Qi, from qualitative history to quantitative measurement," Journal of Alternative and Complementary Medicine, vol. 13, no. 10, pp. 1059-1070, 2007.
[2] C. Y. Chiang, C. T. Chang, H. L. Chu, and L. F. Yang, "Peripheral afferent pathway for acupuncture analgesia," Scientia Sinica, pp. 210-217, 1973.

[3] J. Kong, D. T. Fufa, A. J. Gerber et al., "Psychophysical outcomes from a randomized pilot study of manual, electro, and sham acupuncture treatment on experimentally induced thermal pain," Journal of Pain, vol. 6, no. 1, pp. 55-64, 2005.

[4] W. Takeda and J. Wessel, "Acupuncture for the treatment of pain of osteoarthritic knees," Arthritis Care and Research, vol. 7, no. 3, pp. 118-122, 1994.

[5] A. Benham and M. I. Johnson, "Could acupuncture needle sensation be a predictor of analgesic response?" Acupuncture in Medicine, vol. 27, no. 2, pp. 65-67, 2009.

[6] M. I. Johnson and A. E. Benham, "Acupuncture needle sensation: the emerging evidence," Acupuncture in Medicine, vol. 28, no. 3, pp. 111-114, 2010.

[7] D. C. E. Lim, "What do we know about needling sensation (de qi) and pain outcomes?" Focus on Alternative and Complementary Therapies, vol. 16, no. 2, pp. 126-127, 2011.

[8] P. White, P. Prescott, and G. Lewith, "Does needling sensation (de qi) affect treatment outcome in pain? Analysis of data from a larger single-blind, randomised controlled trial," Acupuncture in Medicine, vol. 28, no. 3, pp. 120-125, 2010.

[9] C. S. Yin, H. J. Park, S. Y. Kim et al., "Electroencephalogram changes according to the subjective acupuncture sensation," Neurological Research, vol. 32, supplement 1, pp. S31-S36, 2010.

[10] J. Vas, E. Perea-Milla, C. Méndez et al., "Efficacy and safety of acupuncture for chronic uncomplicated neck pain: a randomised controlled study," Pain, vol. 126, no. 1-3, pp. 245-255, 2006.

[11] C. Witt, B. Brinkhaus, S. Jena et al., "Acupuncture in patients with osteoarthritis of the knee: a randomised trial," The Lancet, vol. 366, no. 9480, pp. 136-143, 2005.

[12] B. M. Berman, L. Lao, P. Langenberg, W. L. Lee, A. M. K. Gilpin, and M. C. Hochberg, "Effectiveness of acupuncture as 
adjunctive therapy in osteoarthritis of the knee. A randomized, controlled trial," Annals of Internal Medicine, vol. 141, no. 12, pp. 901-910, 2004.

[13] H. P. Scharf, U. Mansmann, K. Streitberger et al., "Acupuncture and knee osteoarthritis: a three-armed randomized trial," Annals of Internal Medicine, vol. 145, no. 1, pp. 12-20, 2006.

[14] M. Haake, H. H. Müller, C. Schade-Brittinger et al., "German Acupuncture Trials (GERAC) for chronic low back pain: randomized, multicenter, blinded, parallel-group trial with 3 groups," Archives of Internal Medicine, vol. 167, no. 17, pp. 18921898, 2007.

[15] A. Benham, G. Phillips, and M. I. Johnson, "An experimental study on the self-report of acupuncture needle sensation during deep needling with bi-directional rotation," Acupuncture in Medicine, vol. 28, no. 1, pp. 16-20, 2010.

[16] K. K. S. Hui, E. E. Nixon, M. G. Vangel et al., "Characterization of the "deqi" response in acupuncture," BMC Complementary and Alternative Medicine, vol. 7, article 33, 2007.

[17] A. Y. Leung, J. Park, G. Schulteis, J. R. Duann, and T. Yaksh, “The electrophysiology of De Qi sensations," Journal of Alternative and Complementary Medicine, vol. 12, no. 8, pp. 743-750, 2006.

[18] H. MacPherson and A. Asghar, "Acupuncture needle sensations associated with De Qi: a classification based on experts' ratings," Journal of Alternative and Complementary Medicine, vol. 12, no. 7, pp. 633-637, 2006.

[19] J. J. Mao, J. T. Farrar, K. Armstrong, A. Donahue, J. Ngo, and M. A. Bowman, "De qi: Chinese acupuncture patients' experiences and beliefs regarding acupuncture needling sensationan exploratory survey," Acupuncture in Medicine, vol. 25, no. 4, pp. 158-165, 2007.

[20] D. Pach, C. Hohmann, R. Lüdtke, F. Zimmermann-Viehoff, C. M. Witt, and C. Thiele, "German translation of the southampton needle sensation questionnaire: use in an experimental acupuncture study," Forschende Komplementarmedizin, vol. 18, no. 6, pp. 321-326, 2011.

[21] J. Park, H. Park, H. Lee, S. Lim, K. Ahn, and H. Lee, "Deqi sensation between the acupuncture-experienced and the Naïve: a Korean study II," American Journal of Chinese Medicine, vol. 33, no. 2, pp. 329-337, 2005.

[22] J. Park, A. White, C. Stevinson, E. Ernst, and M. James, "Validating a new non-penetrating sham acupuncture device: two randomised controlled trials," Acupuncture in Medicine, vol. 20, no. 4, pp. 168-174, 2002.

[23] C. A. Vincent, P. H. Richardson, J. J. Black, and C. E. Pither, “The significance of needle placement site in acupuncture," Journal of Psychosomatic Research, vol. 33, no. 4, pp. 489-496, 1989.

[24] A. White, M. Cummings, P. Barlas et al., "Defining an adequate dose of acupuncture using a neurophysiological approach-a narrative review of the literature," Acupuncture in Medicine, vol. 26, no. 2, pp. 111-120, 2008.

[25] P. White, F. Bishop, H. Hardy et al., "Southampton needle sensation questionnaire: development and validation of a measure to gauge acupuncture needle sensation," Journal of Alternative and Complementary Medicine, vol. 14, no. 4, pp. 373-379, 2008.

[26] D. T. W. Yu, A. Y. M. Jones, and M. Y. C. Pang, "Development and validation of the chinese version of the massachusetts general hospital acupuncture sensation scale: an exploratory and methodological study," Acupuncture in Medicine, vol. 30, no. 3, pp. 214-221, 2012.

[27] K. Zhou, J. Fang, X. Wang et al., "Characterization of De Qi with electroacupuncture at acupoints with different properties,"
Journal of Alternative and Complementary Medicine, vol. 17, no. 11, pp. 1007-1013, 2011.

[28] L. Bai, W. Qin, J. Tian et al., "Acupuncture modulates spontaneous activities in the anticorrelated resting brain networks," Brain Research, vol. 1279, pp. 37-49, 2009.

[29] L. Bai, J. Tian, C. Zhong et al., "Acupuncture modulates temporal neural responses in wide brain networks: evidence from fMRI study," Molecular Pain, vol. 6, article 73, 2010.

[30] R. P. Dhond, C. Yeh, K. Park, N. Kettner, and V. Napadow, "Acupuncture modulates resting state connectivity in default and sensorimotor brain networks," Pain, vol. 136, no. 3, pp. 407418, 2008.

[31] W. Qin, J. Tian, L. Bai et al., "FMRI connectivity analysis of acupuncture effects on an amygdala-associated brain network," Molecular Pain, vol. 4, article 55, 2008.

[32] J. Fang, Z. Jin, Y. Wang et al., "The salient characteristics of the central effects of acupuncture needling: limbic-paralimbicneocortical network modulation," Human Brain Mapping, vol. 30, no. 4, pp. 1196-1206, 2009.

[33] K. K. S. Hui, J. Liu, O. Marina et al., “The integrated response of the human cerebro-cerebellar and limbic systems to acupuncture stimulation at ST 36 as evidenced by fMRI," NeuroImage, vol. 27, no. 3, pp. 479-496, 2005.

[34] V. Napadow, N. Makris, J. Liu, N. W. Kettner, K. K. Kwong, and K. K. S. Hui, "Effects of electroacupuncture versus manual acupuncture on the human brain as measured by fMRI," Human Brain Mapping, vol. 24, no. 3, pp. 193-205, 2005.

[35] S. S. Yoo, E. K. Teh, R. A. Blinder, and F. A. Jolesz, "Modulation of cerebellar activities by acupuncture stimulation: evidence from fMRI study," NeuroImage, vol. 22, no. 2, pp. 932-940, 2004.

[36] J. Sun, W. Qin, M. Dong et al., "Evaluation of group homogeneity during acupuncture stimulation in fMRI studies," Journal of Magnetic Resonance Imaging, vol. 32, no. 2, pp. 298-305, 2010.

[37] L. A. Henderson, R. Bandler, S. C. Gandevia, and V. G. MacEfield, "Distinct forebrain activity patterns during deep versus superficial pain," Pain, vol. 120, no. 3, pp. 286-296, 2006.

[38] F. Beissner, R. Deichmann, C. Henke, and K.-J. Bär, "Acupuncture-deep pain with an autonomic dimension?" NeuroImage, vol. 60, no. 1, pp. 653-660, 2012.

[39] F. Beissner, "Functional magnetic resonance imaging studies of acupuncture mechanisms: a critique," Focus on Alternative and Complementary Therapies, vol. 16, no. 1, pp. 3-11, 2011.

[40] J. Sun, Y. Zhu, L. Jin et al., "Partly separated activations in the spatial distribution between de-qi and sharp pain during acupuncture stimulation: an fMRI-based study," EvidenceBased Complementary and Alternative Medicine, vol. 2012, Article ID 934085, 11 pages, 2012.

[41] T. Lewis, Pain, McMillan, New York, NY, USA, 1942.

[42] R. Bandler, J. L. Price, and K. A. Keay, "Brain mediation of active and passive emotional coping," Progress in Brain Research, vol. 122, pp. 333-349, 2000.

[43] B. M. Lumb, "Hypothalamic and Midbrain Circuitry That Distinguishes between Escapable and Inescapable Pain," News in Physiological Sciences, vol. 19, no. 1, pp. 22-26, 2004.

[44] P. W. Nathan, M. C. Smith, and A. W. Cook, "Sensory effects in man of lesions of the posterior columns and of some other afferent pathways," Brain, vol. 109, no. 5, pp. 1003-1041, 1986.

[45] H. J. W. Nauta, V. M. Soukup, R. H. Fabian et al., "Punctate midline myelotomy for the relief of visceral cancer pain," Journal of Neurosurgery, vol. 92, no. 2, pp. 125-130, 2000. 
[46] W. Noordenbos and P. D. Wall, "Diverse sensory functions with an almost totally divided spinal cord. A case of spinal cord transection with preservation of part of one anterolateral quadrant," Pain, vol. 2, no. 2, pp. 185-195, 1976.

[47] A. C. N. Chen, M. Shimojo, P. Svensson, and L. Arendt-Nielsen, "Brain dynamics of scalp evoked potentials and current source densities to repetitive (5-pulse train) painful stimulation of skin and muscle: central correlate of temporal summation," Brain Topography, vol. 13, no. 1, pp. 59-72, 2000.

[48] L. A. Henderson, S. C. Gandevia, and V. G. Macefield, "Gender differences in brain activity evoked by muscle and cutaneous pain: a retrospective study of single-trial fMRI data," NeuroImage, vol. 39, no. 4, pp. 1867-1876, 2008.

[49] D. M. Niddam, T. Graven-Nielsen, L. Arendt-Nielsen, and A. C. N. Chen, "Non-painful and painful surface and intramuscular electrical stimulation at the thenar and hypothenar sites: differential cerebral dynamics of early to late latency SEPs," Brain Topography, vol. 13, no. 4, pp. 283-292, 2001.

[50] D. M. Niddam, T. C. Yeh, Y. T. Wu et al., "Event-related functional MRI study on central representation of acute muscle pain induced by electrical stimulation," NeuroImage, vol. 17, no. 3, pp. 1437-1450, 2002.

[51] M. Shimojo, P. Svensson, L. Arendt-Nielsen, and A. C. N. Chen, "Dynamic brain topography of somatosensory evoked potentials and equivalent dipoles in response to graded painful skin and muscle stimulation," Brain Topography, vol. 13, no. 1, pp. 43-58, 2000.

[52] P. Svensson, A. Beydoun, T. J. Morrow, and K. L. Casey, "Nonpainful and painful stimulation of human skin and muscle: analysis of cerebral evoked potentials," Electroencephalography and Clinical Neurophysiology_Evoked Potentials, vol. 104, no. 4, pp. 343-350, 1997.

[53] A. U. Asghar, G. Green, M. F. Lythgoe, G. Lewith, and H. MacPherson, "Acupuncture needling sensation: the neural correlates of deqi using fMRI," Brain Research, vol. 1315, pp. 111-118, 2010.

[54] K. K. S. Hui, J. Liu, N. Makris et al., "Acupuncture modulates the limbic system and subcortical gray structures of the human brain: evidence from fMRI studies in normal subjects," Human Brain Mapping, vol. 9, no. 1, pp. 13-25, 2000.

[55] K. K. S. Hui, O. Marina, J. D. Claunch et al., "Acupuncture mobilizes the brain's default mode and its anti-correlated network in healthy subjects," Brain Research, vol. 1287, pp. 84103, 2009.

[56] M. T. Wu, J. M. Sheen, K. H. Chuang et al., "Neuronal specificity of acupuncture response: a fMRI study with electroacupuncture," NeuroImage, vol. 16, no. 4, pp. 1028-1037, 2002.

[57] G. Li, L. Huang, R. T. F. Cheung, S. R. Liu, Q. Y. Ma, and E. S. Yang, "Cortical activations upon stimulation of the sensorimotor-implicated acupoints," Magnetic Resonance Imaging, vol. 22, no. 5, pp. 639-644, 2004.

[58] G. Li, C. R. Jack, and E. S. Yang, "An fMRI study of somatosensory-implicated acupuncture points in stable somatosensory stroke patients," Journal of Magnetic Resonance Imaging, vol. 24, no. 5, pp. 1018-1024, 2006.

[59] D. D. Dougherty, J. Kong, M. Webb, A. A. Bonab, A. J. Fischman, and R. L. Gollub, "A combined [11C]diprenorphine PET study and fMRI study of acupuncture analgesia," Behavioural Brain Research, vol. 193, no. 1, pp. 63-68, 2008.

[60] L. Li, H. Liu, Y. Z. Li et al., "The human brain response to acupuncture on same-meridian acupoints: evidence from an fMRI study," Journal of Alternative and Complementary Medicine, vol. 14, no. 6, pp. 673-678, 2008.

[61] G. Li and E. S. Yang, "An fMRI study of acupuncture-induced brain activation of aphasia stroke patients," Complementary Therapies in Medicine, vol. 19, supplement 1, pp. S49-S59, 2011.

[62] S. Shukla, A. Torossian, J. R. Duann, and A. Leung, "The analgesic effect of electroacupuncture on acute thermal pain perception-a central neural correlate study with fMRI," Molecular Pain, vol. 7, article 45, 2011.

[63] J. L. Fang, T. Krings, J. Weidemann, I. G. Meister, and A. Thron, "Functional MRI in healthy subjects during acupuncture: different effects of needle rotation in real and false acupoints," Neuroradiology, vol. 46, no. 5, pp. 359-362, 2004.

[64] S. M. Wang, R. T. Constable, F. S. Tokoglu, D. A. Weiss, D. Freyle, and Z. N. Kain, "Acupuncture-induced blood oxygenation leveldependent signals in awake and anesthetized volunteers: a pilot study," Anesthesia and Analgesia, vol. 105, no. 2, pp. 499-506, 2007.

[65] W. Wang, L. Liu, X. Zhi et al., "Study on the regulatory effect of electro-acupuncture on Hegu point (LI4) in cerebral response with functional magnetic resonance imaging," Chinese Journal of Integrative Medicine, vol. 13, no. 1, pp. 10-16, 2007.

[66] Y. Zhou and J. Jia, "Effect of acupuncture given at the HT 7, ST 36, ST 40 and KI 3 acupoints on various parts of the brains of Alzheimer's disease patients," Acupuncture and Electro-Therapeutics Research, vol. 33, no. 1-2, pp. 9-17, 2008.

[67] S. U. Park, A. S. Shin, G. H. Jahng, S. K. Moon, and J. M. Park, "Effects of scalp acupuncture versus upper and lower limb acupuncture on signal activation of blood oxygen level dependent (BOLD) fMRI of the brain and somatosensory cortex," Journal of Alternative and Complementary Medicine, vol. 15, no. 11, pp. 1193-1200, 2009.

[68] J. H. Zhang, J. Li, X. D. Cao, and X. Y. Feng, "Can electroacupuncture affect the sympathetic activity, estimated by skin temperature measurement? A functional MRI study on the effect of needling at GB 34 and GB 39 on patients with pain in the lower extremity," Acupuncture and Electro-Therapeutics Research, vol. 34, no. 3-4, pp. 151-164, 2009.

[69] S. Yeo, I. H. Choe, M. Van Den Noort, P. Bosch, and S. Lim, "Consecutive acupuncture stimulations lead to significantly decreased neural responses," Journal of Alternative and Complementary Medicine, vol. 16, no. 4, pp. 481-487, 2010.

[70] S. Yeo, S. Lim, I.-H. Choe et al., "Acupuncture stimulation on gb34 activates neural responses associated with parkinson's disease," CNS Neuroscience and Therapeutics, vol. 18, no. 9, pp. 781-790, 2012.

[71] J. Kong, F. Li, R. Li et al., "A pilot study of functional magnetic resonance imaging of the brain during manual and electroacupuncture stimulation of acupuncture point (LI-4 $\mathrm{Hegu}$ ) in normal subjects reveals differential brain activation between methods," Journal of Alternative and Complementary Medicine, vol. 8, no. 4, pp. 411-419, 2002.

[72] K. Li, B. Shan, J. Xu et al., "Changes in fMRI in the human brain related to different durations of manual acupuncture needling," Journal of Alternative and Complementary Medicine, vol. 12, no. 7, pp. 615-623, 2006.

[73] V. Napadow, N. Kettner, J. Liu et al., "Hypothalamus and amygdala response to acupuncture stimuli in carpal tunnel syndrome," Pain, vol. 130, no. 3, pp. 254-266, 2007.

[74] H. MacPherson, G. Green, A. Nevado et al., "Brain imaging of acupuncture: comparing superficial with deep needling," Neuroscience Letters, vol. 434, no. 1, pp. 144-149, 2008. 
[75] V. Napadow, R. Dhond, K. Park et al., "Time-variant fMRI activity in the brainstem and higher structures in response to acupuncture," NeuroImage, vol. 47, no. 1, pp. 289-301, 2009.

[76] V. Napadow, R. P. Dhond, J. Kim et al., "Brain encoding of acupuncture sensation-coupling on-line rating with fMRI," NeuroImage, vol. 47, no. 3, pp. 1055-1065, 2009.

[77] V. Napadow, J. Lee, J. Kim et al., "Brain correlates of phasic autonomic response to acupuncture stimulation: an eventrelated fMRI study," Human Brain Mapping, 2012.

[78] S. S. Jeun, J. S. Kim, B. S. Kim et al., "Acupuncture stimulation for motor cortex activities: a 3T fMRI Study," American Journal of Chinese Medicine, vol. 33, no. 4, pp. 573-578, 2005.

[79] J. Kong, R. L. Gollub, J. M. Webb, J. T. Kong, M. G. Vangel, and K. Kwong, "Test-retest study of fMRI signal change evoked by electroacupuncture stimulation," NeuroImage, vol. 34, no. 3, pp. 1171-1181, 2007.

[80] B. J. Na, G. H. Jahng, S. U. Park et al., "An fMRI study of neuronal specificity of an acupoint: electroacupuncture stimulation of Yanglingquan (GB34) and its sham point," Neuroscience Letters, vol. 464, no. 1, pp. 1-5, 2009.

[81] L. Bai, H. Yan, N. Li et al., "Neural specificity of acupuncture stimulation at pericardium 6: evidence from an fMRI study," Journal of Magnetic Resonance Imaging, vol. 31, no. 1, pp. 71-77, 2010.

[82] W. Q. Qiu, J. Claunch, J. Kong et al., “The effects of acupuncture on the brain networks for emotion and cognition: an observation of gender differences," Brain Research, vol. 1362, pp. 56-67, 2010.

[83] J. Sun, W. Qin, L. Jin et al., "Impact of global normalization in fMRI acupuncture studies," Evidence-Based Complementary and Alternative Medicine, vol. 2012, Article ID 467061, 22 pages, 2012.

[84] J. H. Zhang, X. D. Cao, J. Li, W. J. Tang, H. Q. Liu, and X. Y. Feng, "Neuronal specificity of needling acupoints at same meridian: a control functional magnetic resonance imaging study with electroacupuncture," Acupuncture and ElectroTherapeutics Research, vol. 32, no. 3-4, pp. 179-193, 2007.

[85] J. Fang, X. Wang, H. Liu et al., "The limbic-prefrontal network modulated by electroacupuncture at CV4 and CV12," EvidenceBased Complementary and Alternative Medicine, vol. 2012, Article ID 515893, 11 pages, 2012.

[86] W. Huang, D. Pach, V. Napadow et al., "Characterizing acupuncture stimuli using brain imaging with fMRI-a systematic review and meta-analysis of the literature," PLoS ONE, vol. 7, no. 4, Article ID e32960, 2012.

[87] V. G. Macefield, S. Gandevia, and L. A. Henderson, "Discrete changes in cortical activation during experimentally induced referred muscle pain: a single-trial fMRI study," Cerebral Cortex, vol. 17, no. 9, pp. 2050-2059, 2007.

[88] L. Maeda, M. Ono, T. Koyama et al., "Human brain activity associated with painful mechanical stimulation to muscle and bone," Journal of Anesthesia, vol. 25, no. 4, pp. 523-530, 2011.

[89] G. K. Aguirre, E. Zarahn, and M. D’Esposito, “The inferential impact of global signal covariates in functional neuroimaging analyses," NeuroImage, vol. 8, no. 3, pp. 302-306, 1998.

[90] A. E. Desjardins, K. A. Kiehl, and P. F. Liddle, "Removal of confounding effects of global signal in functional MRI analyses," NeuroImage, vol. 13, no. 4, pp. 751-758, 2001.

[91] M. Gavrilescu, M. E. Shaw, G. W. Stuart, P. Eckersley, I. D. Svalbe, and G. F. Egan, "Simulation of the effects of global normalization procedures in functional MRI," NeuroImage, vol. 17, no. 2, pp. 532-542, 2002.
[92] M. Junghöfer, H. T. Schupp, R. Stark, and D. Vaitl, "Neuroimaging of emotion: empirical effects of proportional global signal scaling in fMRI data analysis," NeuroImage, vol. 25, no. 2, pp. 520-526, 2005.

[93] F. Beissner and C. Henke, "Methodological problems in fMRI studies on acupuncture: a critical review with special emphasis on visual and auditory cortex activations," Evidence-Based Complementary and Alternative Medicine, vol. 2011, Article ID 607637, 7 pages, 2011.

[94] R. A. Poldrack, "The future of fMRI in cognitive neuroscience," Neuroimage, vol. 62, no. 12, pp. 1216-1220, 2011.

[95] K. Murphy, R. M. Birn, D. A. Handwerker, T. B. Jones, and P. A. Bandettini, "The impact of global signal regression on resting state correlations: are anti-correlated networks introduced?" NeuroImage, vol. 44, no. 3, pp. 893-905, 2009.

[96] G. K. Aguirre, E. Zarahn, and M. D’Esposito, "Empirical analyses of BOLD fMRI statistics. II. Spatially smoothed data collected under null-hypothesis and experimental conditions," NeuroImage, vol. 5, no. 3, pp. 199-212, 1997.

[97] J. L. R. Andersson, "How to estimate global activity independent of changes in local activity," NeuroImage, vol. 6, no. 4, pp. 237244, 1997.

[98] P. M. Macey, K. E. Macey, R. Kumar, and R. M. Harper, "A method for removal of global effects from fMRI time series," NeuroImage, vol. 22, no. 1, pp. 360-366, 2004. 


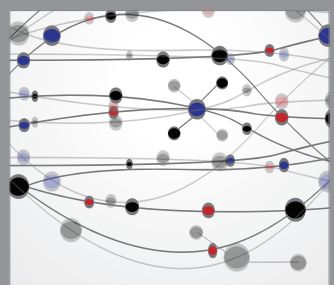

The Scientific World Journal
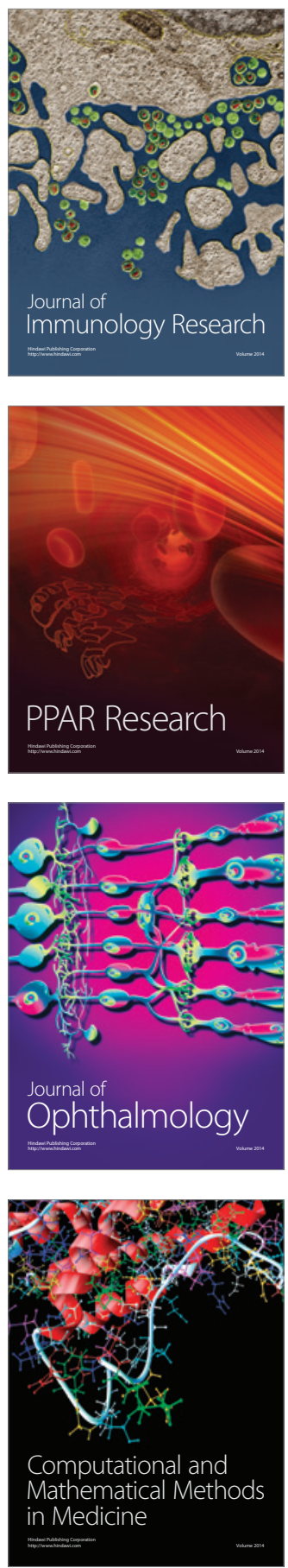

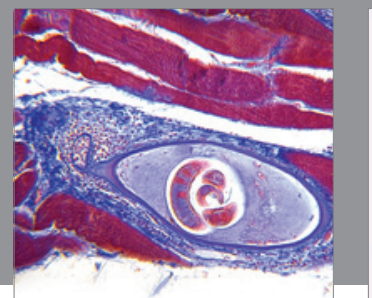

Gastroenterology

Research and Practice
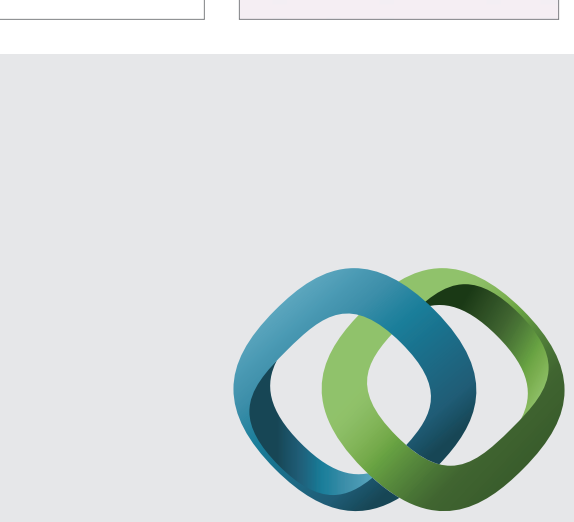

\section{Hindawi}

Submit your manuscripts at

http://www.hindawi.com
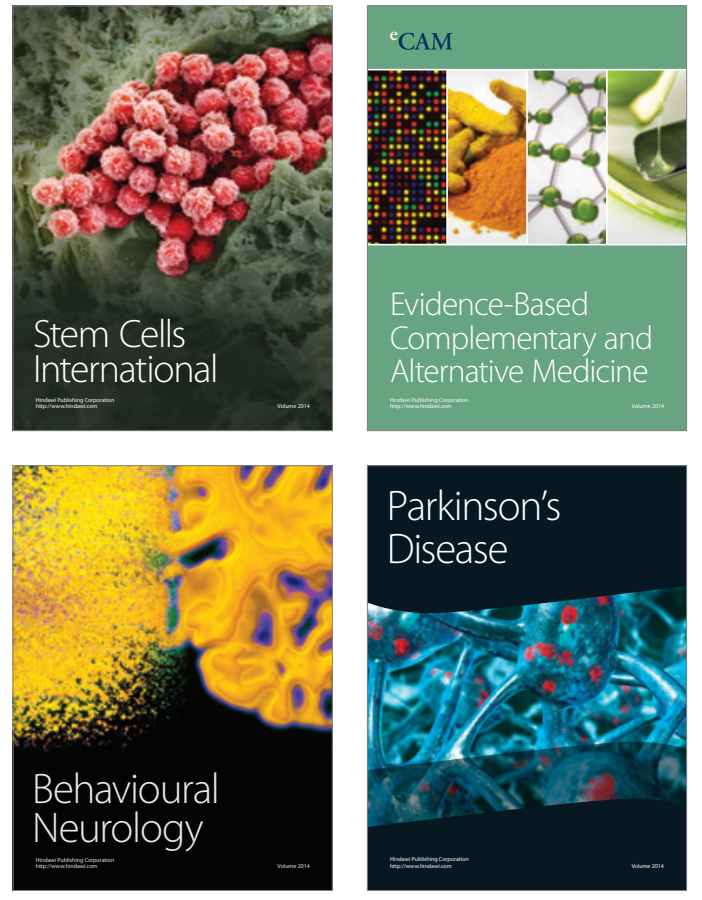
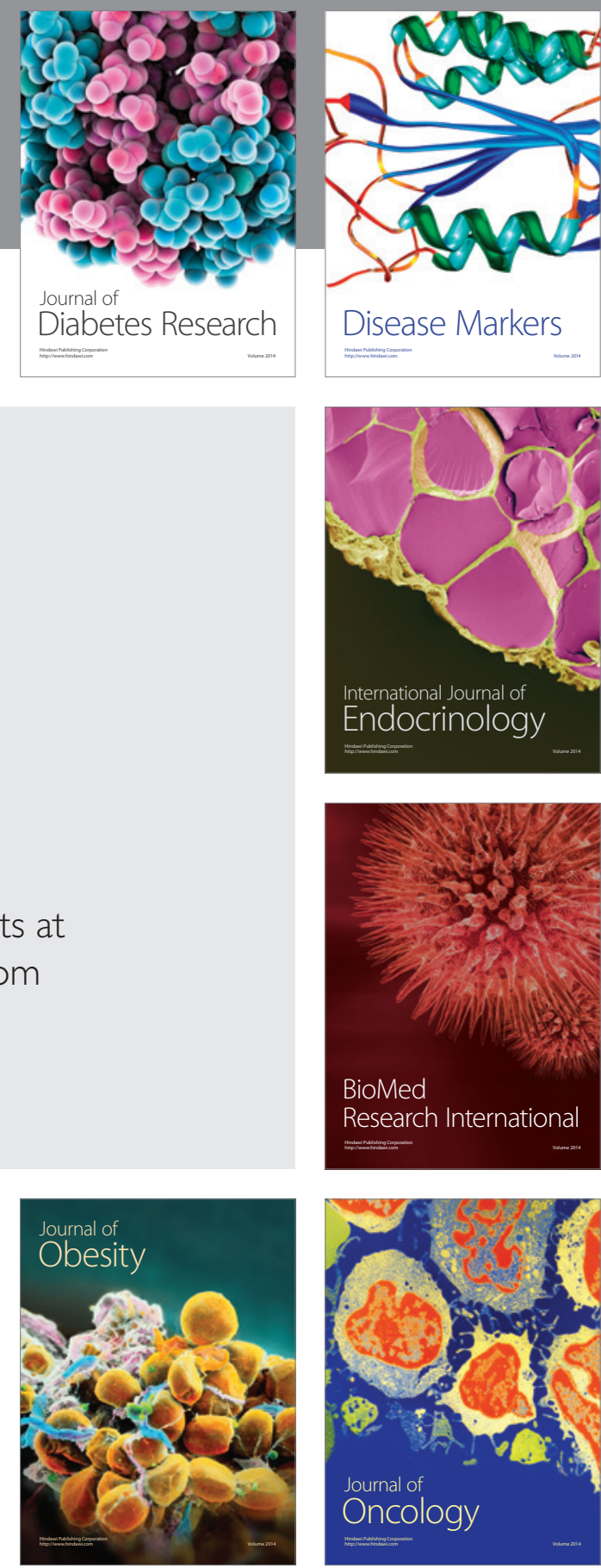

Disease Markers
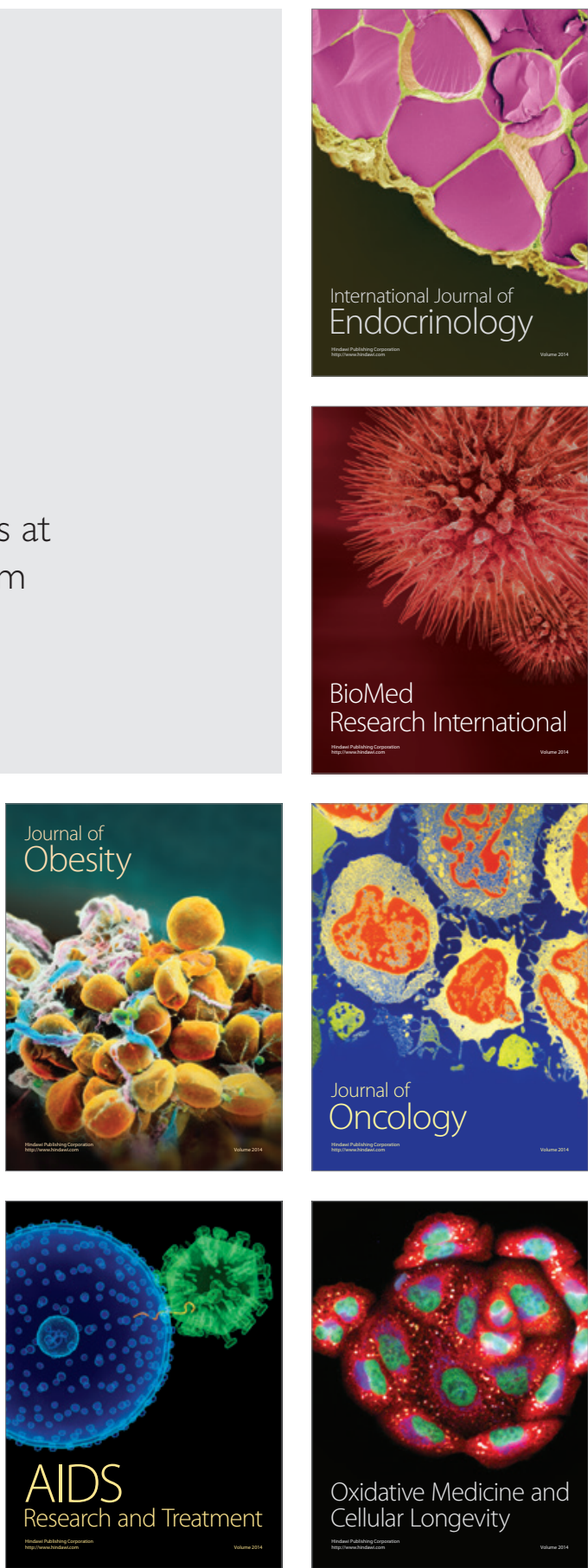